\title{
Healing of human Achilles tendon ruptures: Radiodensity reflects mechanical properties
}

\author{
Thorsten Schepull and Per Aspenberg
}

Linköping University Post Print

\section{Tweet}

N.B.: When citing this work, cite the original article.

The original publication is available at www.springerlink.com:

Thorsten Schepull and Per Aspenberg, Healing of human Achilles tendon ruptures: Radiodensity reflects mechanical properties, 2015, Knee Surgery, Sports Traumatology, Arthroscopy, (23), 3, 884-889.

http://dx.doi.org/10.1007/s00167-013-2720-8

Copyright: Springer Verlag (Germany) http://www.springerlink.com/?MUD=MP

Postprint available at: Linköping University Electronic Press

http://urn.kb.se/resolve?urn=urn:nbn:se:liu:diva-91725 


\section{Healing of human Achilles tendon ruptures: Radiodensity reflects mechanical properties}

Thorsten Schepull, MD, Per Aspenberg, MD PhD

Orthopedics, Department of Clinical and Experimental Medicine, Faculty of Health Sciences, Linköping University, SE 58185 Linköping, Sweden

*Address correspondence to Thorsten Schepull, MD, Orthopedics, Department of Clinical and Experimental Medicine, Faculty of Health Sciences, Linköping University, SE 58185

Linköping, Sweden

e-mail: thorsten.schepull@lio.se 


\begin{abstract}
Purpose

We investigated if radiodensity from computed tomography can be used to quantitatively evaluate the healing of ruptured Achilles tendons.

Methods
\end{abstract}

We measured the radiodensity of the healing tendons in 65 patients who were treated for Achilles tendon rupture, and tested the hypothesis that density would correlate with an estimate for e-modulus, derived from strain, measured by Roentgen Stereophotogrammetric Analysis (RSA), with different mechanical loading.

Results

Radiodensity 7 weeks after injury was decreased to $67 \%$ of the contralateral, uninjured tendon. There was no improvement in radiodensity from 7 to 19 weeks, whereas at one year it had increased to $106 \%$. Only 2 of 52 measured values at one year were lower than the highest value at 19 weeks, i.e. there was minimal overlap. The variation in radiodensity could explain $80 \%$ of the variation in e-modulus, but radiodensity correlated only weakly with e-modulus at each time point separately. At one year, both radiodensity and e-modulus correlated with functional results, although weakly.

\title{
Conclusions
}

From 19 weeks onwards, radiodensity appears to reflect mechanical properties of the tendon and might to some extent predict the final outcome. Radiodensity at 7 weeks is difficult to interpret, probably because it reflects both callus and damaged tissues.

\section{Level of evidence}




\section{Key Words}

Density, CT, modulus of elasticity, mechanical properties 


\section{Introduction}

Rupture of the Achilles tendon leads to a long period of rehabilitation and a long period before patients regain full physical activity. To analyze the healing process, clinical findings and various scores and questionnaires are used (Leppilahti et al. 1998; Nilsson-Helander et al. 2007; Thermann et al. 1995). Still, we have little information about the material properties of the Achilles tendon during the repair process.

If we had a means of quantitatively estimating the quality of the repairing tendon at a given time point, this could be used both to compare treatment methods and to advice individual patients an appropriate loading activity. In recent times, ultrasound (US) and magnetic resonance imaging (MRI) have been used for diagnosing ruptures of the Achilles tendon (Bleakney et al. 2002; Khan et al. 2003; Majewski et al. 2003; Schweitzer 1993). US has also been used to monitor the healing process, quantifying the thickness and structure of the tendon but not the quality of the repair process (Majewski, Lehmann 2003; Thermann et al. 2002). However, to our knowledge, there is no previous study using radiodensity as measured by computed tomography (CT) for evaluating the quality of the healing Achilles tendon. With CT one can quantify radiodensity in Hounsfield units (H-Units). The Hounsfield scale is a quantitative scale. Sir Godfrey Newbold Hounsfield defined $0 \mathrm{H}$-Units as the radiodensity of distilled water at standard pressure and temperature, and -1000 H-Units as the radiodensity of air (Hounsfield 1980).

The rationale for using CT is that tendons can be roughly described to consist of collagen and water. Also the tissue formed during repair - the tendon callus - consists of mostly collagen and water, although the higher cellularity and looser matrix organisation gives it a higher water content than the normal tendon (Kjaer 2004). Tendons have a higher radiodensity than water due to the collagen content. Because water has a radiodensity of $0 \mathrm{H}$-Units, any material 
consisting of water and some other uniform substance would have a radiodensity directly proportional to the concentration of this substance. If tendons were regarded as consisting of collagen and water only, any change in radiodensity would correspond to a similar change in collagen/water ratio. We wanted to study the change in radiodensity and transverse area during the repair of the Achilles tendon, in the hope of finding a quantitative variable that could indicate the quality of the healing Achilles tendon. Thus our hypothesis was that there would be a significant correlation between radiodensity and e-modulus. 


\section{Material and Methods}

We have reanalyzed data from 2 previously performed, randomized trials, in which CT was used for measurement of transverse area and for confirmation of the implantation site of tantalum beads, which were inserted in the tendon as markers for estimation of the stressstrain relationship by use of a Roentgen Stereophotogrammetric analysis (RSA). One trial that has been published (Schepull et al. 2011) (here named study 1), compared operated tendons with or without the addition of a platelet concentrate. No significant differences between the groups were found. The other study (submitted manuscript) examined the effect of early, controlled loading on the healing of the tendon (named study 2). In this study, patients exposed to early loading showed an increased elastic modulus and stiffness of the tendon 19 weeks and one year after injury, but no other differences compared to patients who were immobilized. All patients from these studies were included in the present CT-analysis. In both studies, all patients between 18 and 60 years of age presenting with an acute rupture of the Achilles tendon at our hospital were asked to participate, and almost all eligible consented. Both studies had been approved by the regional ethics board in Linköping, Sweden (Approval \# M81-07 and \# M192-08).

For estimation of the error of measurement of the RSA method for estimating modulus of elasticity, we pooled study 1 with an older study ( $\underline{\mathbf{R E F} \text { op-kons }}$ ), because only these two studies comprised double measurements.

Exclusion criteria were previous Achilles tendon injury, diabetes mellitus, a history of cancer or lung or heart diseases, or any other diseases that could compromise the locomotor system. For details see the original papers. Between September 2007 and April 2008 (study 1) and February 2009 and October 2011 (study 2), we included 65 patients. Their mean age was 41 
(range $24-59$ ) years. There were 11 women. The number of patients with complete examinations at 7, 19 and 52 weeks was 61,58 and 53 respectively.

\section{Operative and postoperative treatment}

In all patients, surgery was done using a conventional open technique with a dorsomedial approach. We used local anaesthesia with Carbocaine (mepivacaine hydrochloride) and adrenaline. The ends of the tendon were adapted with a resorbable suture (Vicryl size 0) using the single-loop Kessler technique. We implanted 2 tantalum beads (size, $0.8 \mathrm{~mm}$ ) in the distal part of the tendon and 2 tantalum beads of the same size in the proximal part.

All patients in study 1 and all control patients in study 2 wore a short-leg cast, with the foot in the equinus position, for 3.5 weeks. After 3.5 weeks, the cast was replaced with a new one in which the ankle was held in neutral position. Full weight-bearing was allowed as tolerated from the beginning. The cast was removed after 7 weeks in total.

The patients in the treatment group in study 2 had their cast removed after 2 weeks and were instead provided with a foam walker including heel wedges. Heel wedges were used to maintain the equinus position, similar to the control group. The wedges were removed gradually during the treatment period. The foam walker was removed after 7 weeks, in order to have an immobilisation period of the same length as in the control group. During the last 5 weeks, the patients were allowed to remove the foam walker twice a day and used a special training device. 
Full weight-bearing on the cast or foam walker was allowed as tolerated from the beginning for all patients in both studies.

All patients started with physiotherapy after the cast or foam walker was removed, i.e. after 7 weeks. At that time point, all patients received the same instructions for home training from the same physiotherapist at our hospital. They were then referred to physiotherapists uninvolved in this research. The physiotherapists were instructed to follow the regional rehabilitation guidelines for Achilles tendon ruptures. Full activity, including sports, was allowed after approximately 5 months.

\section{$\underline{\text { CT evaluation }}$}

In both studies, CT was performed at 7, 19 and 52 weeks after the rupture. Radiodensity was not included in the previous publications for reasons of space and clarity, and because we wanted to combine data from both studies for a separate analysis.

The radiodensity of the tendon was measured in H-units using a circular region of interest in the centre of the Achilles tendon. Measurements were done on sections transverse to the tendon on different levels at the rupture site. The region of interest, i.e. the rupture site, could be identified due to the fact that 2 Tantalum beads had been implanted distally to the rupture and 2 beads proximally to the rupture during surgery. The measurement used for analysis was taken at an equal distance from the proximal and distal beads (Figure 1).

Moreover, computed tomography (CT) was used to measure the transverse area of the tendon at the rupture site. All CT measurements were performed at 7, 19, and 52 weeks after injury. 


\section{Mechanical properties and functional outcome}

To calculate e-modulus, RSA was used to measure strain under defined loading conditions, and computed tomography (CT) was used to measure the transverse area of the tendon at the rupture site (see above). RSA uses small metal beads inserted in the tissue, and enables precise measurements of the distance between them by using simultaneous biplane radiographs and a calibration cage. Data are analysed with specific software. RSA measurements were performed at 7,19 and 52 weeks. At these follow-ups, the patients sat on an examination table with the foot in a specially designed frame which allowed defined dorsiflexion moments to be applied to the ankle. The patients were asked to keep the foot in position and to resist this moment during loading with different loads.

At 7 and 19 weeks we used the same RSA protocol. After a preloading with $150 \mathrm{~N}$ and a 3 minute rest, a $25 \mathrm{~N}$ force was applied, and the first $\mathrm{x}$-rays were taken. After an additional 3 minute rest a $150 \mathrm{~N}$ force was applied. The $25-\mathrm{N}$ loading was intended to provide a baseline value, whereas $150 \mathrm{~N}$ was the main loading. The patients had to resist the force for 15 seconds before the radiographs were taken; thereafter, the weight was immediately removed. Strainper-force values (assuming a linear relationship) were calculated with correction for the lever arms of the forefoot and the calcaneus.

The final RSA was done 12 months after surgery. This examination differed slightly from the first 2 examinations. Also at this time, the patients had to resist the applied loading for 15 seconds with 3-minute intervals, but we increased the force from the baseline $25 \mathrm{~N}$ in $200-\mathrm{N}$ increments, with no repetition (i.e., the forces were 25, 225 and 425 N). Strain per force was now calculated from the slope of the regression lines for all measurements. 
We used the RSA software (UmRSA 4.1 system, RSA Biomedical, Umea, Sweden) to calculate distances between single beads. Tendon force was calculated from pedal force. The pedal pivoted around an axis so that the force had a defined loading point in a lateral projection. Lever arms were calculated from CT lateral radiographs, with the center of the talar trochlea as pivot point (pedal point to trochlea center, trochlea center to center of tendon).

\section{Follow-up: Functional outcome}

We assessed functional results for 56 patients at the 1-year follow-up. All patients were examined by one physiotherapist and the uninjured side was always tested first.

Muscle performance was evaluated with maximal number of single-limb heel raises (cadence, 30 raises per minute; height of the heel should be at least $5 \mathrm{~cm}$ from the floor) and maximal height of a single-limb heel raise, measured in centimeters from the floor to the heel. We used a heel-raise index, defined as the number of heel raises that the patient could do, multiplied by height of the single heel raise and normalized as a percentage of the other side (Schepull et al. 2007). Tests that combine the number of heel raises and their height have been found to have good reliability and validity in detecting functional impairments (Olsson et al. 2012;

Silbernagel et al. 2010). All patients also filled in and returned the form for the validated Achilles Tendon Total rupture Score (ATRS) (Nilsson-Helander, Thomee 2007). The ATRS has been shown to have good validity, reliability and sensitivity for limitations related to symptoms during various activities in individuals who have had total ruptures of the Achilles tendon.

\section{$\underline{\text { Statistical analysis }}$}


Regression between radiodensity and elastic modulus for the entire material (all time points) involves the use of dependent data. To circumvent this problem, and still use all values to get an overall regression coefficient, we used a regression analysis with indicator variables according to the formula $Y=b_{0}+b_{1} X+b_{2} Z_{1}+b_{3} Z_{2}+b_{4} X Z_{1}+b_{5} X Z_{2}$

, where $\mathrm{Y}$ is Hounsfield units, $\mathrm{X}$ is modulus, $\mathrm{Z} 1$ is 1 for 19 weeks otherwise 0 and $\mathrm{Z} 2$ is 1 for 52 weeks otherwise 0.

A simple linear regression was used when comparing values for radiodensity and elastic modulus at the same time point and for comparing data from a single time point with clinical outcome measures at one year. The error of measurement for elastic modulus was derived from double measurements. The correlation between radiodensity and "true" elastic modulus was calculated by removing the influence of the error of measurement according to the formula

$\operatorname{cor}[\mathrm{Y}, \mathrm{X}]=\operatorname{cor}[\mathrm{Y}, \mathrm{X}+\mathrm{e}] / \mathrm{sqrt}(\mathrm{Rx})$, where $\mathrm{Rx}=\operatorname{Var}[\mathrm{X}] /(\operatorname{Var}[\mathrm{X}]+\operatorname{Var}[\mathrm{e}])$

$\mathrm{Y}$ is Hounsfield units, $\mathrm{X}$ is "true" modulus and e is the error of measurement. 


\section{Results}

We included 65 patients with a main age of 40 years (range $24-59$ years). 11 patients were female. There were no complications related to the implantation of the tantalum beads.

3 patients were excluded early on, due to infection, rerupture and pain syndrome. Complete data for radiodensity and e-modulus were available for 61 patients at 7 weeks, 58 patients at 19 weeks, and for 53 at one year. In total, complete data (all time points, including functional outcome) was available for 47 patients.

\section{$\underline{\text { Radiodensity over time }}$}

The radiodensity within the rupture site 7 weeks after injury was decreased to 67 (sd 11) \% of that of the non-injured contralateral tendon. The absolute value was $48 \mathrm{HU}$ (sd 5) compared to the non-injured side $73 \mathrm{HU}$ (sd 11). The density remained low at 19 weeks (50 HU, sd 6). At one year, density was restored (75 HU, sd 7) (Figure 2). There was minimal overlap between 19 weeks and one year: only 2 of 52 values at one year were lower than the highest value at 19 weeks (Figure 3).

The variation within the measurement area of each tendon was expressed as the standard deviation of all pixels within the area. This standard deviation was $15 \%$ of the average density after 7 weeks, $13 \%$ after 19 weeks, and $10 \%$ after one year. The density appeared homogenous within the rupture site between the Tantalum beads, and also farther from the injury i. e. the values for Hounsfield units were similar along a large part of the tendon.

\section{$\underline{\text { Relation between radiodensity and elastic modulus }}$}

The over-all regression analysis (involving all time points) showed that the variation in radiodensity could explain $82 \%$ of the variation in elastic modulus $\left(r^{2}=0.82\right)$. 
The radiodensity at 7 weeks did not correlate with e-modulus at 7 weeks or functional outcome at one year. In contrast, radiodensity at 19 weeks and one year correlated with emodulus at the same point of time, and also with Heel-Raise-Index at one year. However, in contrast to the over-all analysis, the correlations for single time points were weak. As this could be due to a large measuring error in the single estimation of elastic modulus, we calculated the correlation between radiodensity and "true" elastic modulus, as described above.

The measuring error at 19 weeks (sd for difference between the double measurements $\mathrm{x} 2^{-0.5}$ ) was $28 \mathrm{MPa}$, which corresponds to $29 \%$ of the mean of the second measurement. Therefore, the correlation between radiodensity and "true" elastic modulus was considerably stronger than between the measured values ( 19 weeks: $\left.r^{2}=0.23\right)$. This suggests that the radiodensity reflects a quarter of the mechanical properties also at a single time point.

We noted that correlations between Hounsfield units and e-modulus at 19 weeks in study 2 were weaker than in study 1 . We therefore repeated the analysis of the 19 weeks data for patients who had all been treated in a plaster (i.e. we excluded the loaded group in study 2). This analysis showed stronger correlations between measured e-modulus and density $\left(\mathrm{r}^{2}=\right.$ 0.26 ; $\mathrm{p}<0.001)$, whereas in the loaded group, there was no significant correlation at all $\left(\mathrm{r}^{2}=\right.$ $0.01)$

\section{$\underline{\text { Relation between radiodensity and functional results }}$}

The radiodensity at 7 weeks showed no significant correlation with heel-raise index or ATRS. At 19 weeks there was a weak but significant correlation with heel-raise index $\left(r^{2}=0.12 ; p=\right.$ 
0.009). At one year, radiodensity correlated weakly with both heel-raise index and ATRS $\left(\mathrm{r}^{2}=\right.$ $0.17 ; \mathrm{p}=0.002$ and $\left.\mathrm{r}^{2}=0.24 ; \mathrm{p}=0.000\right)$. 


\section{Discussion}

We could confirm the hypothesis that there would be a correlation between radiodensity and e-modulus in healing Achilles tendons. In the over-all analysis, as much as $80 \%$ of the variation in e-modulus could be explained by radiodensity. However, for this is to be practically useful, the method must be able to separate cases also at single time points, and here the correlations were weak. This could in part be explained by a large measuring error of a single estimation of elastic modulus. When this was compensated for, a quarter of the variation in "true" e-modulus could be explained by radiodensity at the same time point. This suggests that radiodensity contains valuable information.

We noted that the correlation between radiodensity and e-modulus was almost absent in the subgroup of patients that had been subjected to mechanical loading. This might be explained by effects of loading. If loading mainly influences collagen orientation, but not density, the emodulus could be expected to increase without a corresponding increase in radiodensity. Indeed, loading increased elastic modulus at 19 weeks by $34 \%$, but radiodensity was only increased by $8 \%$. Therefore, if radiodensity were to be used for group comparisons, effects of differences in rehabilitations programs could be missed.

In contrast, radiodensity could be quite useful for longitudinal studies, as the changes over time from 19 weeks to one year were distinct. Radiodensity values showed hardly any overlap between these time points, whereas there was considerable overlap for elastic modulus (Figure 3).

Earlier studies have shown a clear increase of the Achilles tendon cross-sectional area during the first weeks after removal of the cast, while the e-modulus appears to remain on a constant 
low level during this period (Schepull et al. 2010; Schepull, Kvist 2011). This is apparently reflected by radiodensity, which seems not to increase during the first period of healing. In previous studies, we found a correlation between early e-modulus and late functional results (HRI\%) (Schepull, Kvist 2007; Schepull, Kvist 2010; Schepull, Kvist 2011). Early radiodensity appears not to carry such prognostic information.

It appears that during the first months of healing, the dominating process is callus growth, while improvement of "quality" in terms of better mechanical properties seems to follow in a later phase. This later period of rehabilitation, affecting "quality", seems to be largely finished after one year, as elastic mechanical properties at one year appear similar to normal. The increased risk of rerupture is considered to persist several months after injury. This could perhaps correspond to the period with a low radiodensity.

The risk of rerupture after Achilles tendon injury would be easier to manage if there were a method to quantitate the properties of the healing tendon during the relevant time period. For clinical use, such a method would have to be non-invasive, simple and inexpensive. Computerized tomography fulfils these criteria, and provides quantitative and standardized values for tissue density. The radiation dose is relatively low and does not involve hematopoetic bone marrow. Our study suggests a relation between radiodensity and mechanical properties, but does not address possible clinical utility of this finding. The findings, however, suggests that longitudinal studies with repeated measurements during the remodelling period might give insights into the possibility to use CT for clinical follow-up. As there was almost no overlap between radiodensity measurements at 19 weeks and one year (Figure 3), it is possible that restored radiodensity values could serve as an indicator of "completed" healing, and perhaps that the period with an increased risk of rerupture is over. 


\section{Figure Legends}

Figure 1:

Areas of interest for measurement of Hounsfield Units

\section{Figure 2:}

Radiodensity in Hounsfield Units (HU) at 7, 19 and 52 weeks. All measurements. Values from the same patients are connected with a line

Figure 3:

Hounsfield Units and e-modulus (MPa) at 19 weeks (+) and 52 weeks $(\bullet)$ 


\section{References}

Bleakney RR, Tallon C, Wong JK, Lim KP, Maffulli N. Long-term ultrasonographic features of the Achilles tendon after rupture. Clinical journal of sport medicine : official journal of the Canadian Academy of Sport Medicine. 2002: 12: 273-278.

Hounsfield GN. Nobel Award address. Computed medical imaging. Med Phys. 1980: 7: 283290.

Khan KM, Forster BB, Robinson J, Cheong Y, Louis L, Maclean L, Taunton JE. Are ultrasound and magnetic resonance imaging of value in assessment of Achilles tendon disorders? A two year prospective study. Br J Sports Med. 2003: 37: 149-153.

Kjaer M. Role of extracellular matrix in adaptation of tendon and skeletal muscle to mechanical loading. Physiol Rev. 2004: 84: 649-698.

Leppilahti J, Forsman K, Puranen J, Orava S. Outcome and prognostic factors of achilles rupture repair using a new scoring method. Clinical orthopaedics and related research. 1998: $152-161$.

Majewski M, Lehmann M, Dick W, Steinbruck K. [Value of sonography to monitor the course of Achilles tendon rupture after treatment--comparison of conservative therapy, percutaneous tendon adaptation, and open suture]. Unfallchirurg. 2003: 106: 556-560.

Nilsson-Helander K, Thomee R, Gravare-Silbernagel K, Thomee P, Faxen E, Eriksson BI, Karlsson J. The Achilles tendon Total Rupture Score (ATRS): development and validation. Am J Sports Med. 2007: 35: 421-426.

Olsson N, Karlsson J, Eriksson BI, Brorsson A, Lundberg M, Silbernagel KG. Ability to perform a single heel-rise is significantly related to patient-reported outcome after Achilles tendon rupture. Scand J Med Sci Sports. 2012. 
Schepull T, Kvist J, Andersson C, Aspenberg P. Mechanical properties during healing of Achilles tendon ruptures to predict final outcome: a pilot Roentgen stereophotogrammetric analysis in 10 patients. BMC Musculoskelet Disord. 2007: 8: 116.

Schepull T, Kvist J, Aspenberg P. Early E-modulus of healing Achilles tendons correlates with late function. Similar results with or without surgery. Scand J Med Sci Sports. 2010: In press. Schepull T, Kvist J, Norrman H, Trinks M, Berlin G, Aspenberg P. Autologous platelets have no effect on the healing of human achilles tendon ruptures: a randomized single-blind study. Am J Sports Med. 2011: 39: 38-47.

Schweitzer ME. Magnetic resonance imaging of the foot and ankle. Magn Reson Q. 1993: 9: 214-234.

Silbernagel KG, Nilsson-Helander K, Thomee R, Eriksson BI, Karlsson J. A new measurement of heel-rise endurance with the ability to detect functional deficits in patients with Achilles tendon rupture. Knee Surg Sports Traumatol Arthrosc. 2010: 18: 258-264. Thermann H, Frerichs O, Biewener A, Krettek C, Schandelmeier P. [Functional treatment of acute rupture of the Achilles tendon. An experimental biomechanical study]. Unfallchirurg. 1995: 98: 507-513.

Thermann H, Frerichs O, Holch M, Biewener A. Healing of Achilles tendon, an experimental study: part 2--Histological, immunohistological and ultrasonographic analysis. Foot Ankle Int. 2002: 23: 606-613. 


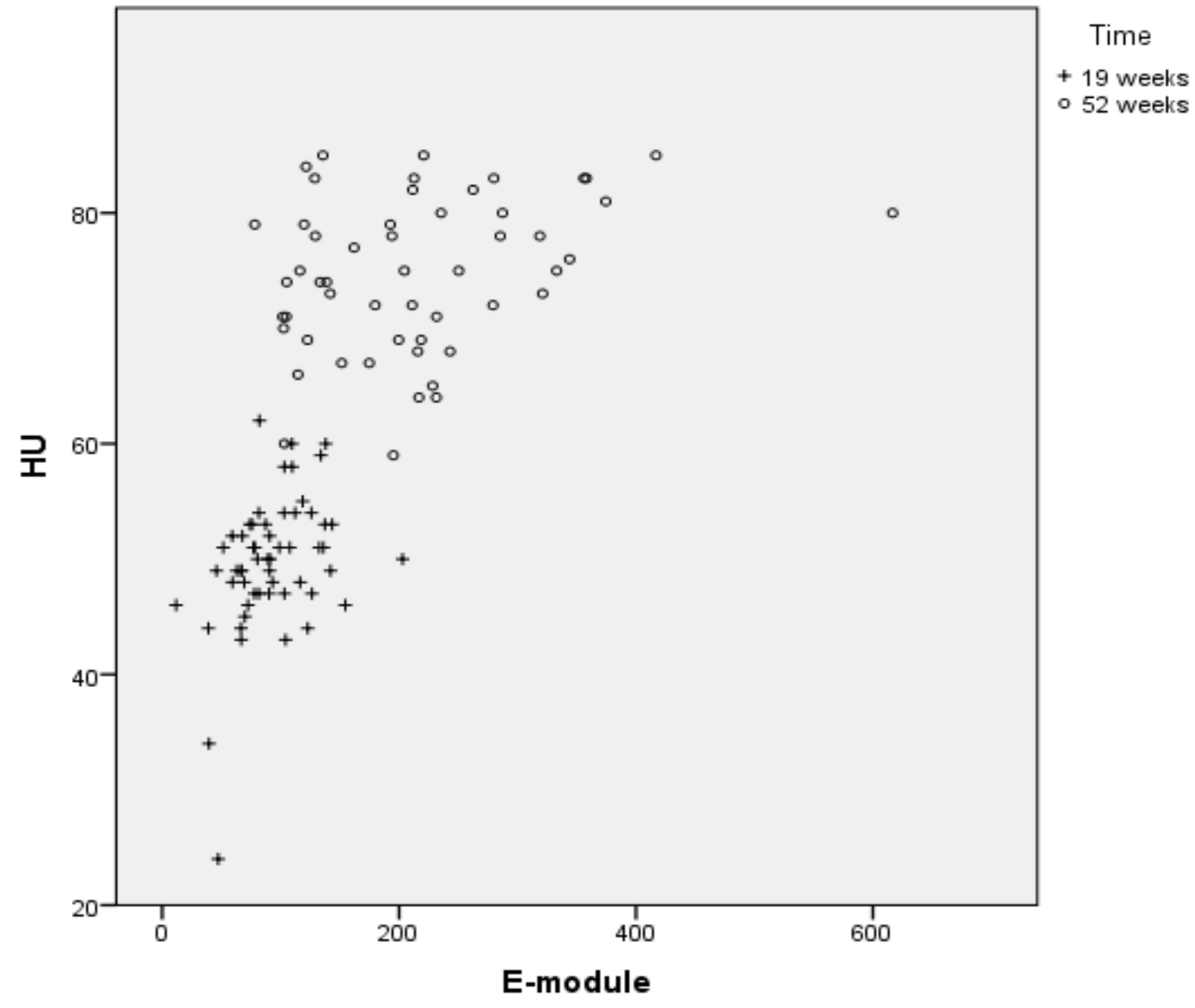

